\title{
A proposal to measure iron opacity at conditions close to the solar convective zone-radiative zone boundary
}

\author{
D J Hoarty ${ }^{1, \text { a) }}$, J Morton ${ }^{1}$, M Jeffery ${ }^{1}$, L K Pattison ${ }^{1}$, A Wardlow ${ }^{1}$, S P D Mangles ${ }^{2}$, \\ S J Rose ${ }^{2}$, C Iglesias ${ }^{3}$, K Opachich ${ }^{4}$, R F Heeter ${ }^{3}$, T S Perry ${ }^{5}$ \\ ${ }^{1} A W E$ plc, Reading, RG7 4PR, UK. \\ ${ }^{2}$ Plasma Physics Group, Blackett Laboratory, Imperial College London, London, SW7 2AZ, UK \\ ${ }^{3}$ Lawrence Livermore National Laboratory, Livermore, CA 94550, USA. \\ ${ }^{4}$ Nevada National Security Site, Livermore, CA, USA \\ ${ }^{5}$ Los Alamos National Laboratory, Los Alamos, New Mexico, NMxxxx, USA \\ a) Corresponding author: David.Hoarty@awe.co.uk
}

\begin{abstract}
A major problem in stellar modelling is the discrepancy between solar models and helioseismology data in the position of the convective zone-radiative zone boundary in the sun. This could be explained by a large uncertainty in the calculated opacity data and recent experimental data on iron using the Sandia National Laboratory Z facility have shown large differences, up to a factor of 4 , between measurement and prediction at plasma conditions close to the convective zone-radiative zone boundary. This paper describes a proposal for a radiative burn-through experiment to be fielded on NIF to observe if a radiation wave transit through a $\mathrm{Fe}_{2} \mathrm{O}_{3}$ sample is consistent with the factor of 2-4 change in the iron opacity seen in the $\mathrm{Z}$ experiments. A target design and the diagnostic method are described. A detailed radiationhydrodynamic model has been used to generate synthetic results and explore the sensitivities and experimental accuracy needed for the proposed measurement.
\end{abstract}

PACS numbers: $52.50 \mathrm{Jm}, 52.25 \mathrm{Jm}, 52.25 \mathrm{Os}, 52.65 \mathrm{Rr}$

Keywords: Plasma radiative opacity; Stellar modelling; Radiative transport 


\section{INTRODUCTION}

As our closest star, the Sun has been intensively studied and is used as a standard to compare to other stars in the wider Universe. A revision of the solar composition has been implemented in the last fifteen years or so due to the work of Asplund and co-workers [1-3] determining the solar elemental abundances of $\mathrm{C}, \mathrm{N}, \mathrm{O}, \mathrm{Ne}$ and $\mathrm{Ar}$, on the surface of the sun, using three-dimensional rather than one-dimensional solar atmospheric models. Although the revised composition answers some anomalies seen in the sun [4] it creates a problem for modelling of the solar interior. Very precise helioseismology techniques $[5,6]$ have determined the radial position of the solar convective zoneradiative zone boundary, where energy transport changes from radiative diffusion to convection, but the value predicted using the revised solar composition differs from these measurements. It has been suggested that this convective zone-radiative zone position problem in the sun could be accounted for by uncertainties in the calculation of the radiative opacity of the solar elements $[4,6,7]$. Recent opacity experiments [8, 9] have recorded a significant difference between the measured and predicted opacity of iron, an important element in determining the radiation transport in the sun, at conditions close to those at the convective-radiative zone boundary, where the temperature is approximately $200 \mathrm{eV}$, and the density $200 \mathrm{mg} / \mathrm{cc}$, corresponding to an electron density $10^{23} / \mathrm{cc}$. These experiments were carried out at the Sandia National Laboratory Z facility and show an increased opacity in iron of between 2-4 times that predicted by theory in the region of the iron L-shell transitions and bound-free edge over the x-ray energy range of $750-1300 \mathrm{eV}$. This large difference remains unexplained and has significant implications for opacity theory since it is in violation of the Thomas-Reiche-Kuhn oscillator sum rule [10]. Since the findings of the $\mathrm{Z}$ experiments were reported an enhancement in the iron opacity has been invoked by several groups to explain pulsation in $\mathrm{O}$ and B-type stars [11, 12]. A change in the opacity of iron has implications in asteroseismology [13] and the calculation of luminosity variation in the search for exoplanets.

There is currently a campaign of experiments at NIF to repeat the iron experiments using similar techniques of Xray heating and point projection radiography as used for the frequency resolved transmission measurements made at the $\mathrm{Z}$ facility [14]. The proposed experiment would use a different and complementary technique in which the transit of a radiation wave through an iron rich sample is used to infer the iron opacity. In the modelling of stellar interiors radiation transfer simulations use the diffusion approximation and the Rosseland mean opacity. The Rosseland mean 
is the harmonic mean opacity weighted by the derivative of the Planck function with temperature and integrated over frequency $[15,16]$ where the peak of the weighting function is at an X-ray energy approximately 4 times the temperature. To investigate the parameters of importance to the propagation of a diffusive radiation wave it is instructive to equate the energy transfer by radiation diffusion per unit area to the energy increase in an element of the material and rearrange to give equation 1 .

$$
x^{2}=\mathrm{A} \cdot \frac{T^{3} t}{\rho^{2} C_{v} \kappa_{R}}
$$

Where $x$ is the propagation distance of the radiation wave; $\mathrm{A}$ is a constant; $T$ is the temperature behind the wavefront; $\rho$ is the density of the sample material through which the wave propagates; $C_{v}$ is the specific heat of the material at constant volume; $t$ is the propagation time and $\kappa_{R}$ is the Rosseland mean opacity.

To infer the opacity from the burn-through of a supersonic, diffusive radiation wave transiting a sample requires accurate knowledge of the parameters of equation 1. For supersonic waves $\rho$ is constant and must be determined from prior target calibration along with target thickness $x . C_{v}$ is obtained from a knowledge of the material equation of state. Most importantly, the temperature or flux driving the front must be accurately measured. As described below, to produce a radiation wave which is supersonic for long enough to transit a sample at a density and temperature relevant to the convective zone-radiative zone boundary, the energy of a NIF or Z class facility is required. NIF also has the diagnostics to accurately measure both the flux driving the radiation front and the emergent flux and the timing of the radiation wave break-out.

\section{EXPERIMENTAL METHOD}

A radiation field relevant to studies of the solar interior can be achieved on NIF using a gold hohlraum to convert the laser energy into a radiation drive. The hohlraum configuration proposed has been used previously on NIF [17] and uses the halfraum design where beams enter through a single laser entry hole (LEH) in a gold cylinder with the sample package mounted on the opposite side of the cylinder. The dimensions of the cylinder are $3.5 \times 3 \mathrm{~mm}$ as shown in the Fig 1. with $25 \mu \mathrm{m}$ thick gold walls and a $2.4 \mathrm{~mm}$ LEH. Fig 1 also shows the schematic layout of the target, beams and diagnostics. In experiments to date using this type of hohlraum 80 beams of $2.5 \mathrm{~ns}$ square pulses, $0.351 \mu \mathrm{m}$ 
wavelength irradiating at $30^{\circ}, 44.5^{\circ}$, and $50^{\circ}$ from the cylinder axis with an energy of $360 \mathrm{~kJ}$ total produced a peak radiation temperature in the hohlraum of $350 \mathrm{eV}$ measured using the NIF DANTE [18] spectrometer, which uses an array of filtered x-ray diodes to give ten channels that record time-resolved x-ray fluxes emitted from the hohlraum viewing through the LEH. The proposed experiment would measure the hohlraum temperature in the same way with a DANTE 1 diagnostic viewing at $64^{\circ}$ to the hohlraum. A $2 \mathrm{~mm}$ diameter, $1.2 \mathrm{~mm}$ long gold cylinder and filled with $150 \mathrm{mg} / \mathrm{cc}$ iron oxide foam is attached to the hohlraum so that the radiation flux from the hohlraum drives a supersonic radiation wave through the foam and along the length of the cylinder to emerge at the rear surface. The arrival time and emergent flux of this radiation wave at the rear of the foam is measured by the second NIF DANTE viewing at $37^{\circ}$ to the cylinder axis. To establish the arrival time accurately a separate laser beam irradiates the outside of the gold cylinder at a known delay after the laser beams used to heat the hohlraum. This produces an $\mathrm{x}$-ray signal that is recorded on the DANTE and can show the timing of the radiation break-out with respect to the heating beams to an accuracy of 100ps. This is a standard technique used at NIF to provide an accurate timing reference for DANTE. Based on previous measurements [17] the uncertainty on the hohlraum drive flux is 5-7\%.

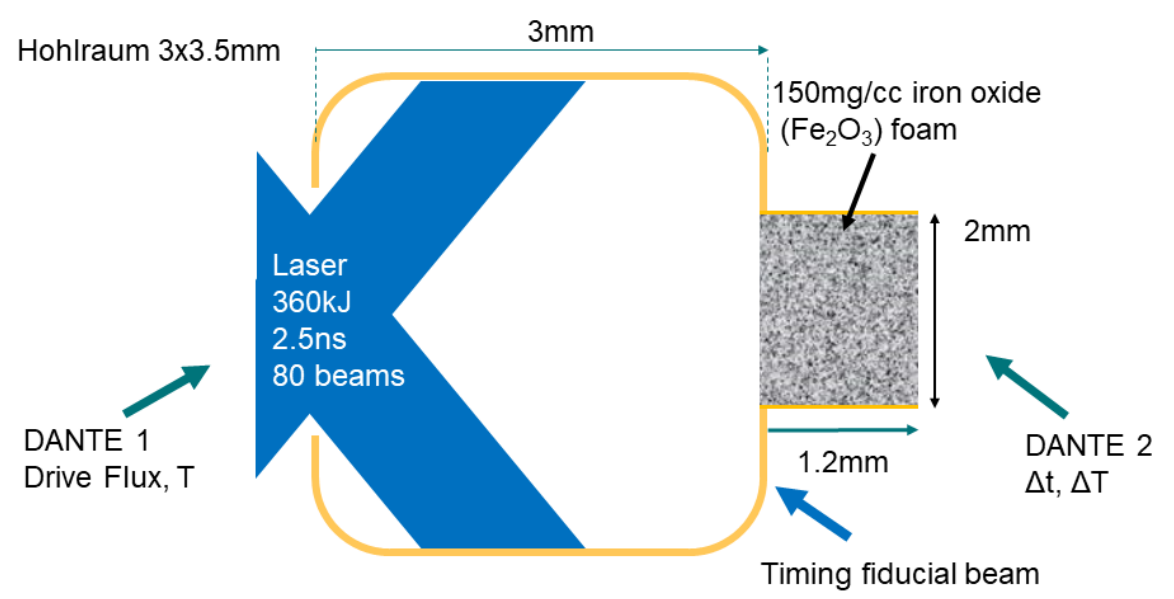

Fig 1. (Colour online) A schematic of the NIF hohlraum and sample package along with the main diagnostics. Again, based on previous work the timing uncertainty is estimated as \pm 100 ps or around $4 \%$. The uncertainty on the foam length is estimated as $3 \%$.

Fig 2 shows example plots of the predicted evolution of the radiation wave profile driven by the hohlraum shown in Fig 1. These are the predictions of the two-dimensional radiation-hydrodynamics code NYM [19]. The plots are the temperatures as a function of position for a $1.4 \mathrm{~mm}$ long foam cylinder. The different curves show the temperature and density profiles of the radiation wave at different times during the wave propagation. The front shows a 
characteristic parabolic profile with a "foot" extending ahead of the main front into the foam [20, 21]. This foot is produced by the higher frequencies in the radiation field which have a longer mean free path in the foam. The wave propagates supersonically through the foam at about Mach 5 so that the foam density is not perturbed on the passage of the wave-front.

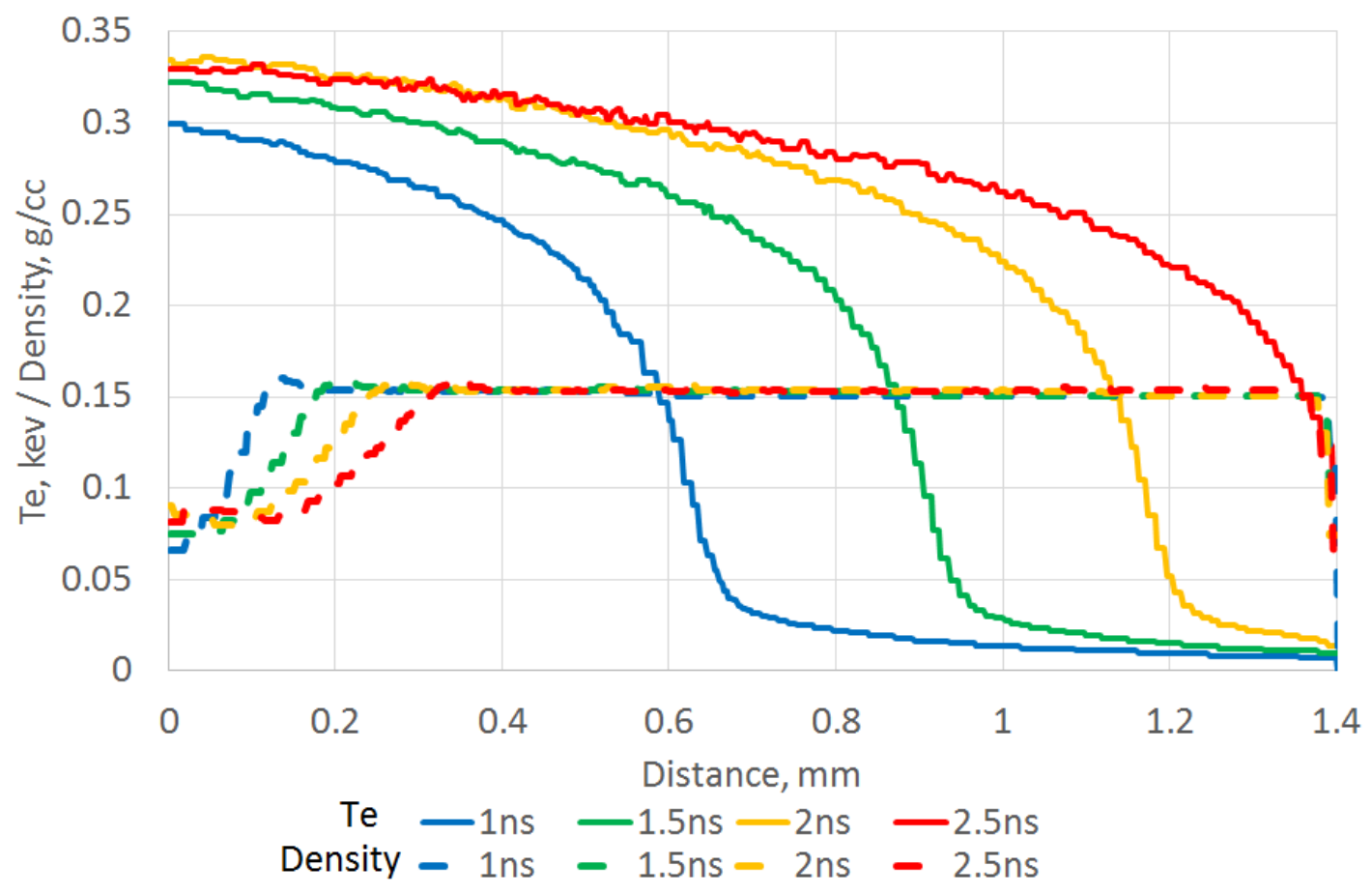

Fig 2. (Colour online) Simulated temperature profiles at different times during the propagation of a radiative wave moving through $150 \mathrm{mg} / \mathrm{cc}$ iron oxide foam.

In Fig 2 the rarefaction wave can be seen at the hohlraum end of the foam cylinder at the time of the break-out of the radiation front at the rear of the foam cylinder. The wave breaks out at about $2.5 \mathrm{~ns}$ for a $1.4 \mathrm{~mm}$ cylinder and between 2 and $2.5 \mathrm{~ns}$ for a $1.2 \mathrm{~mm}$ cylinder. The sample density in the experiment must be sufficiently high that the radiation flow is diffusive and so sensitive to the opacity of iron, but not so high that the wave propagation is subsonic or transonic which complicates the analysis by introducing more uncertainty in establishing the sample density. This precludes the use of solid density iron foil, even on NIF, because of the energy needed to drive a supersonic wave in a solid density iron sample. 
Metal foam technology is available to create iron foam, but oxidation of the iron would make the foam unstable and make it difficult to establish the oxygen content. Instead an iron oxide foam would be used which has the advantage of stability allowing an accurate characterization to be carried out ahead of the experiments. The disadvantage is that the opacity of the oxygen also plays a part in determining the radiation flow. In the solar interior the relative abundance is much greater than in the experimental samples where the transport is dominated overwhelmingly by the iron opacity. In the following predictions described below it is assumed the oxygen opacity is known. The foot ahead of the main wave-front produced by the high frequencies in the radiation field is useful because this heating closes the pores in the foam, in tens of picoseconds, so that the main front propagates through a continuous medium. Iron oxide foams have been manufactured at AWE and characterized by weighing; x-ray tomography at different frequencies; scanning electron microscopy and using combustion analysis to measure elemental composition.

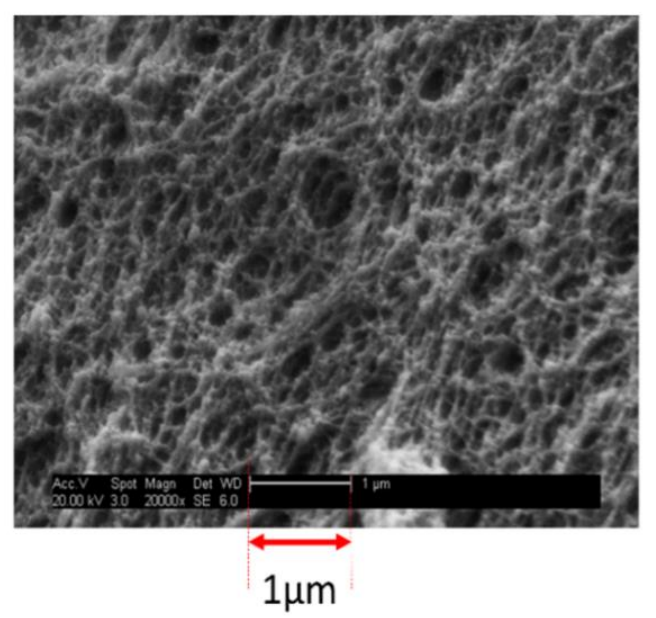

Fig 3: A scanning electron microscope image of the iron oxide foam suggests pore sizes $1-2 \mu \mathrm{m}$ or less. Foam billets are selected based on x-ray tomography.

Fig 3 is an SEM image of the foam showing the pore sizes to be $1-2 \mu \mathrm{m}$ or less. The $\mathrm{x}$-ray tomography allows selection of foam billets from the larger bulk manufactured foam. Experience of previous campaigns using foam targets indicates that the foam density can be established to an accuracy of $\pm 2 \%$. 


\section{PREDICTED RESULTS AND MODELLING}

The target design and the predictions of the radiation wave behaviour are the result of integrated twodimensional radiation-hydrodynamics simulations using the NYM code to model the laser-hohlraum interaction and the radiation wave propagation. The predicted fluxes and timing at the end of the foam cylinder were used to produce a simulated DANTE trace to show the expected experimental data. The effect on simulation prediction of changing the important parameters in the experiment to their error bound limits was also modelled.

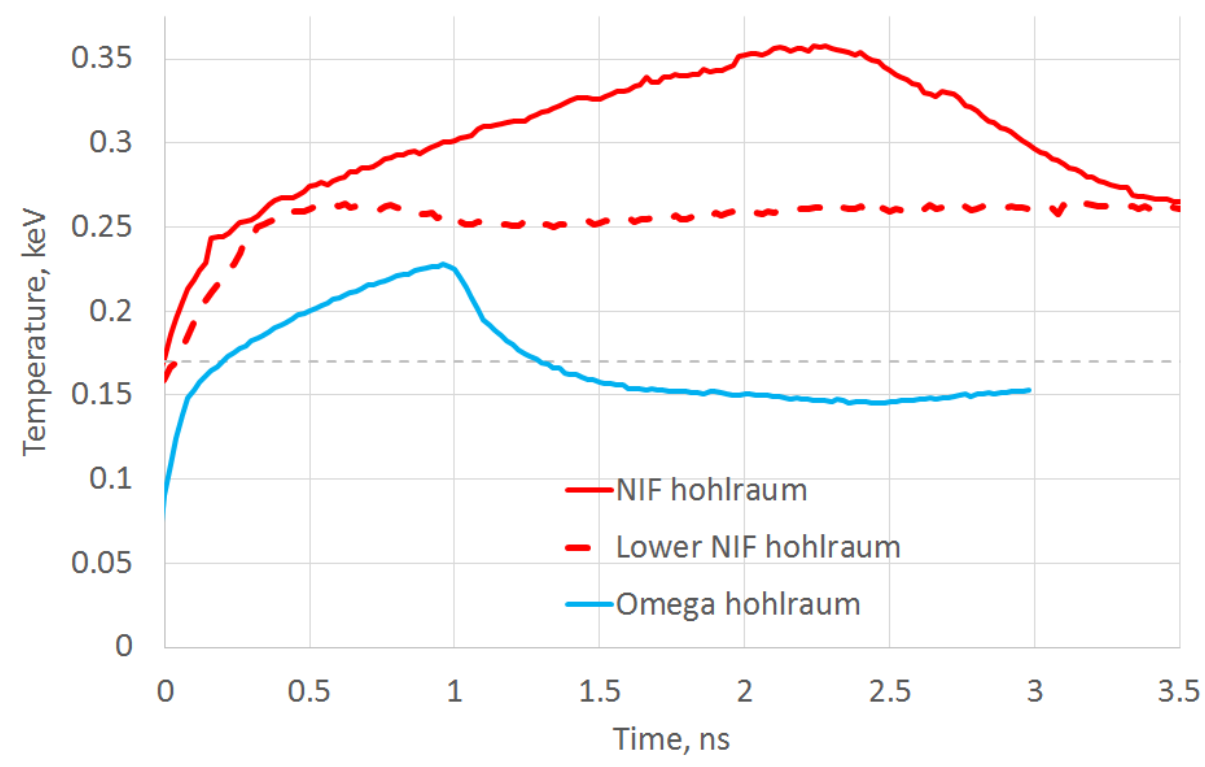

Fig 4. (Colour online) Calculated hohlraum radiation temperature versus time for three cases. The red curve is the drive predicted for the hohlraum shown in Fig. 1 with 80 beams, 2.5ns pulses, 360kJ drive total; the dashed curve is the same hohlraum but driven with 64 beams, $3.5 \mathrm{~ns}$ drive, $196 \mathrm{~kJ}$ total laser energy; the remaining curve is a hohlraum drive achievable on OMEGA with a $1.2 \times 1.6 \mathrm{~mm}$ hohlraum, $5.8 \mathrm{~kJ}$ total energy, $1 \mathrm{~ns}$ pulses.

The simulations resolve the opacity into 288 groups calculated using the state-of-the-art opacity model CASSANDRA [22] with the radiation transport calculated using an Implicit Monte-Carlo method [23]. The equation of state is calculated from tables generated using the NuQEOS [24] model. For the hohlraum-laser interaction the non-local thermodynamic equilibrium plasma properties are calculated using an in-line average-atom screened-hydrogenic model. In Fig. 4 the hohlraum temperature history for three cases is considered. The first case is similar to that characterized in the experiments of reference 20; the second is an alternative drive more tuned to the conditions at the 
CZ boundary and the third is the drive achievable on the OMEGA laser using lower energy and a smaller hohlraum. For the experiment to be sensitive to changes in opacity, at the conditions of interest a transit time of several nanoseconds for a wave at, or above, $200 \mathrm{eV}$ radiation temperature is required which is not possible using the OMEGA hohlraum. The suitability of both NIF drives is considered. Simulated DANTE traces for the higher temperature case are shown in Fig 5.

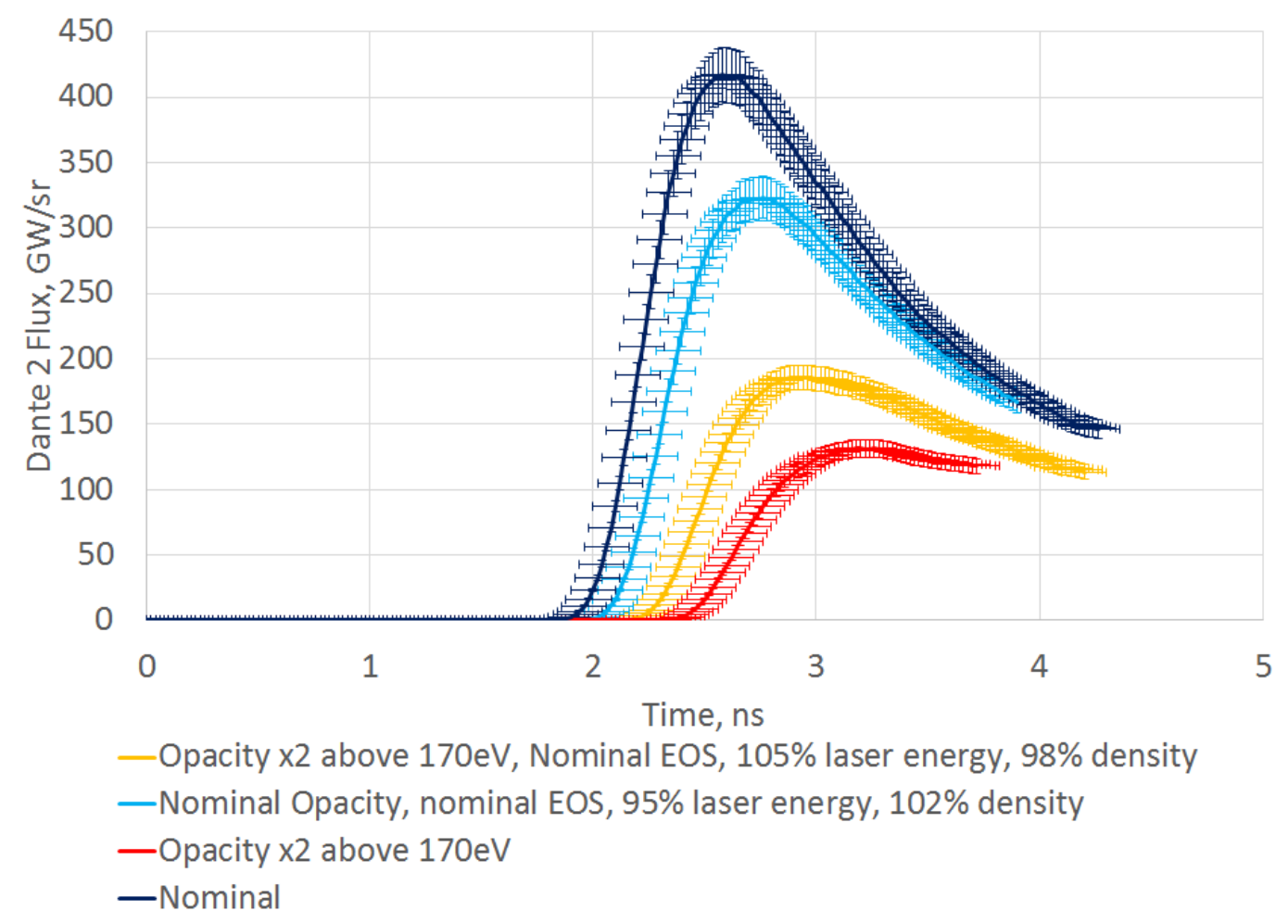

Fig 5. (Colour online) Simulated DANTE results showing the sensitivity to the different parameters determining the radiation wave transit in the $150 \mathrm{mg} / \mathrm{cc}, 1.2 \mathrm{~mm}$ long iron oxide foam. The error bars represent the uncertainty in the DANTE timing $( \pm 100 \mathrm{ps})$ and flux measurement $( \pm 5 \%)$.

Fig. 5 shows the predicted results from the DANTE 2 diagnostic viewing the rear of the foam cylinder at $37^{\circ}$ from the cylinder axis. The largest sensitivity is to the laser drive and hence radiation temperature, as can be seen from equation 1. The curves show the prediction for the break-out time and emergent flux for the nominal opacity, laser energy (radiation flux), foam density and equation of state and the prediction if the iron opacity for temperatures above $170 \mathrm{eV}$ is multiplied by a factor of 2 as implied by the results from the Sandia experiments. There is a $\log -\log$ interpolation between the $165 \mathrm{eV}$ and $170 \mathrm{eV}$ grid points, otherwise temperatures below $165 \mathrm{eV}$ are at nominal opacity 
values in-line with the Sandia data [12]. The increased opacity results in a later break-out and a lower emergent flux. Also shown are the curve at one error limit where iron opacity is increased but the laser energy is $5 \%$ higher than nominal and the density is $2 \%$ lower, both of which will move the break-out earlier. The other error bound limit shown is with the nominal opacity values but with the laser drive at the lower bound, and the density at the high bound both of which move the break-out later. Taking the experimental uncertainties into account in this way, for the experiment to be feasible there must be a measurable difference between the latter curves. Taking the DANTE timing errors into account there is only a small difference in the rise time for the two curves. However, there is a large difference in the emergent flux which is more than an order of magnitude larger than the $8 \mathrm{GW} / \mathrm{sr}$ detection threshold.

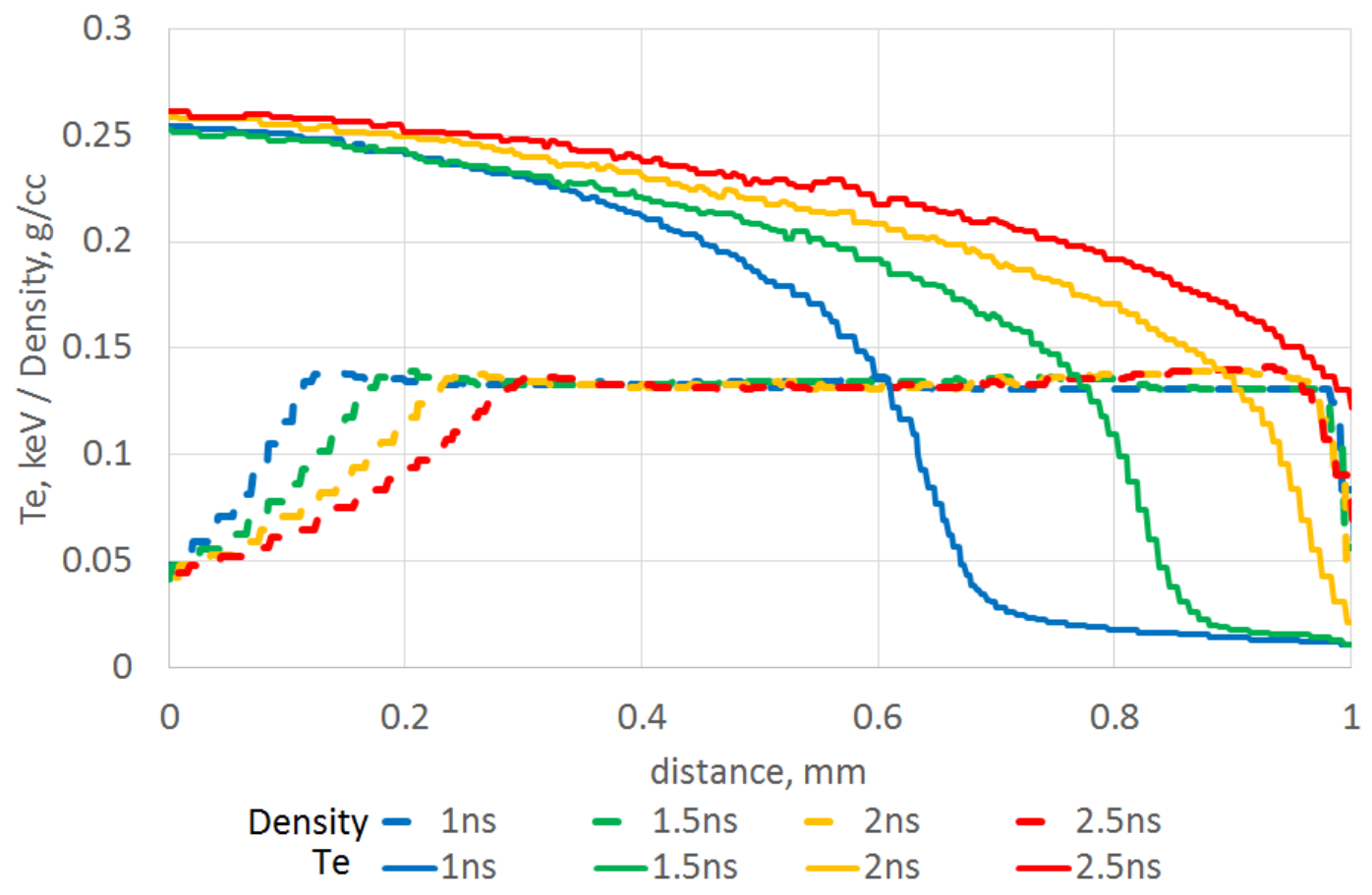

Fig 6: Temperature v distance through a $130 \mathrm{mg} / \mathrm{cc}, 1 \mathrm{~mm}$ long iron oxide foam for a $196 \mathrm{~kJ}, 3.5 \mathrm{~ns}$ drive.

An alternative drive flux could be provided by reducing the hohlraum energy and lengthening the laser pulses as shown in the lower NIF drive of Fig 4. The temperature profiles in this case are shown in Fig 6. the propagation is supersonic and the profiles are similar to the those of the higher drive case. The foam density is adjusted slightly to $130 \mathrm{mg} / \mathrm{cc}$ and the foam is shortened so that the radiation wave remains supersonic up to the point of break-out. The simulated DANTE traces are shown in Fig 7. As before the curves show the effect of changing the opacity at temperatures above $170 \mathrm{eV}$ by a factor of two multiplier and the sensitivity to the uncertainty bounds in laser drive and foam density. In this case there is clear separation in time in the radiation wave break-out when the opacity is changed 
as well as a difference in the emergent flux. Although the signal level is significantly lower than for the higher flux drive shown in Fig 5 this is still many times the $8 \mathrm{GW} / \mathrm{sr}$ detection threshold. The separation of time and emergent flux and the lower temperature in this case suggests that despite the lower signal levels this case is the preferred option for the experiment.

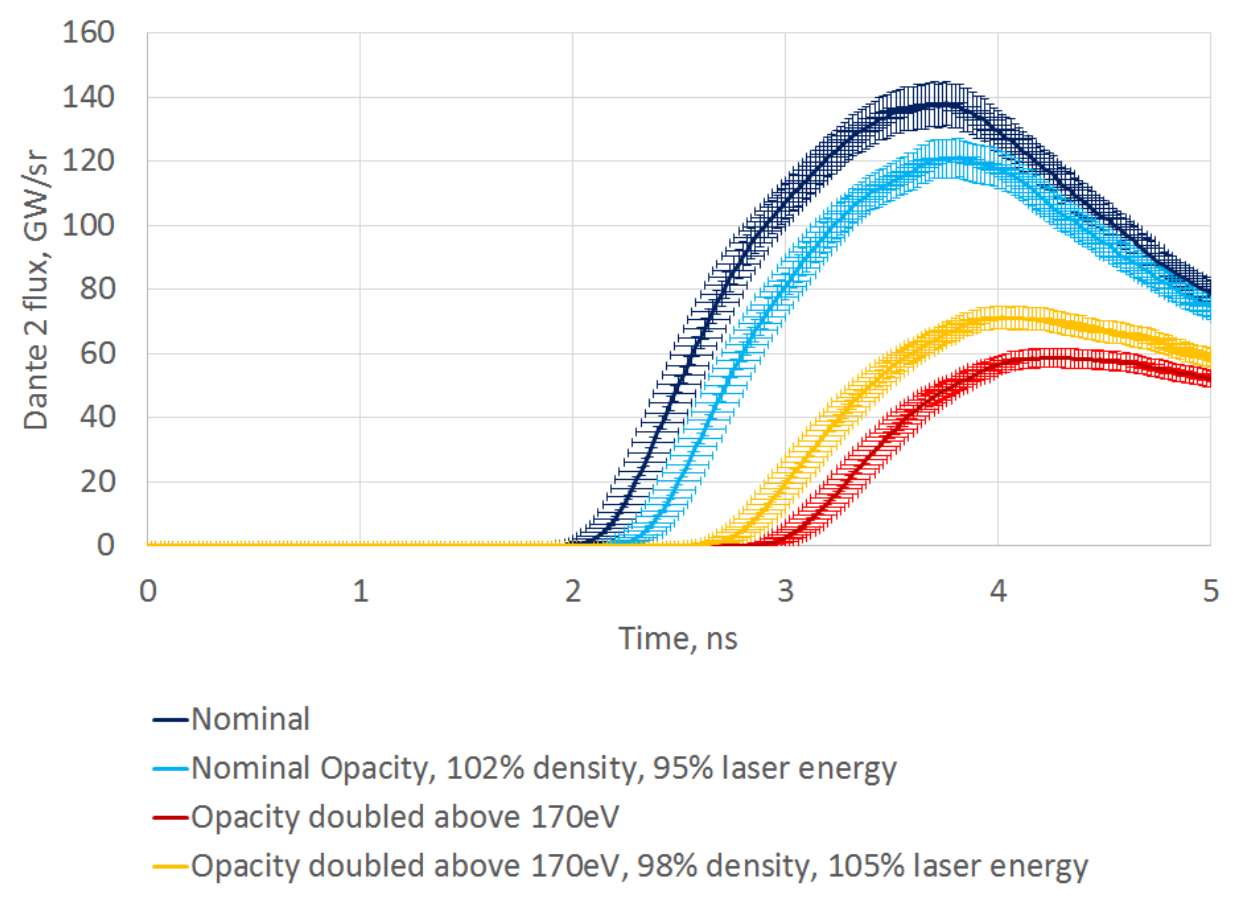

Fig 7: (colour online) Simulation DANTE traces for a hohlraum drive of $196 \mathrm{~kJ}, 3.5 \mathrm{~ns}$ pulses and a $130 \mathrm{mg} / \mathrm{cc}$ foam cylinder $1 \mathrm{~mm}$ long.

A non-thermal component of the radiation field from the gold M-band emission around 2-3keV comes from the laser spots inside the hohlraum. The effect of M-band on the predicted break-out time was simulated by comparing a default radiation field result with one where the radiation emission in the range 1.8-3.6keV was multiplied by a factor of 2 while keeping the overall energy content of the radiation field the same in both cases by also reducing the emission in the range $0.125-1.25 \mathrm{keV}$ to compensate. Though the simulation with the increased M-band did not run beyond 4.2ns the result, plotted in Fig 8, shows the small shift in the timing of the break-out and emergent flux due to the increased energy at higher frequencies. This change is within the error bars of the timing and flux measurement uncertainty. 


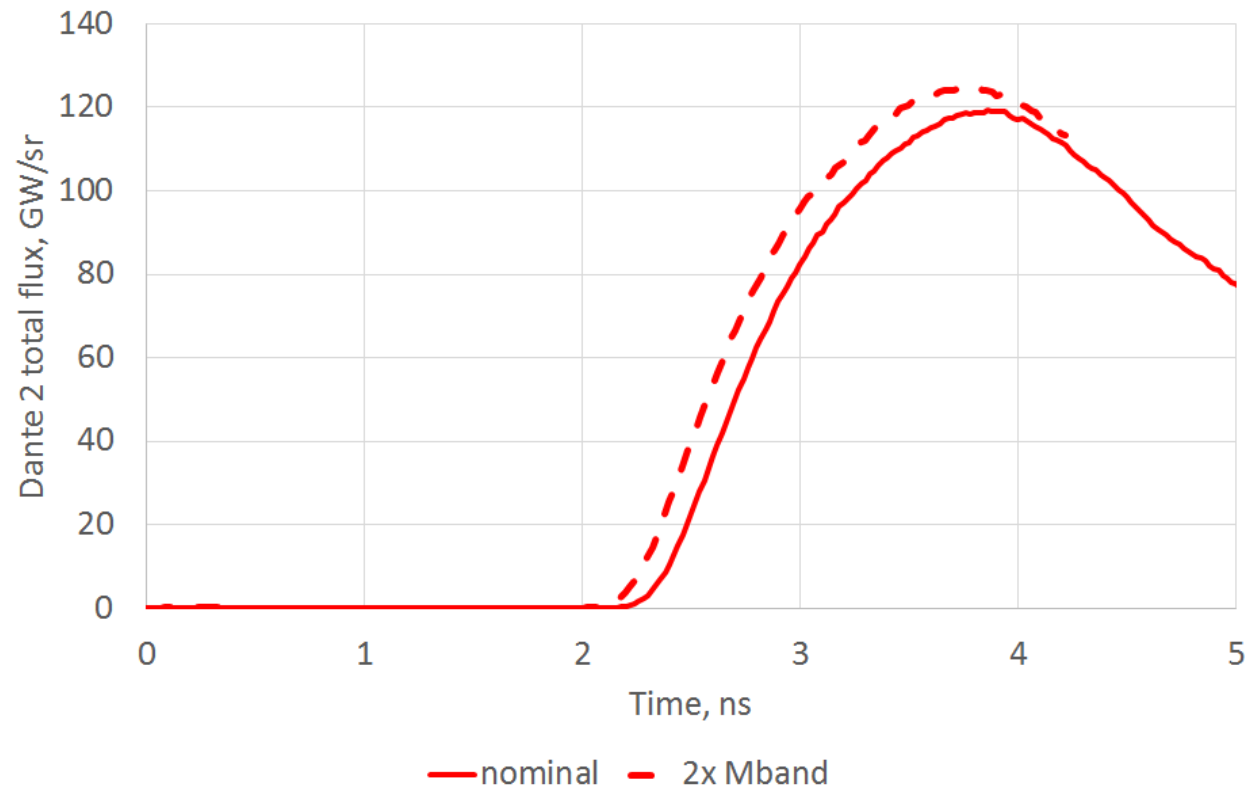

Fig 8. (Color online). Predicted DANTE 2 results for the emergent radiation wave for a doubling of the M band radiation in the radiation field.

A further possible uncertainty is the equation of state of the foam which determines the specific heat (see equation 1). At the densities and temperatures of the experiment the foam would be expected to behave as an ideal plasma where:-

$$
\begin{aligned}
& P=\left(Z^{*}+1\right) n_{i} T \\
& U=\frac{3}{2}\left(Z^{*}+1\right) n_{i} T+\sum_{i} E_{i}
\end{aligned}
$$

$P, T, Z^{*}, n_{i}, U$ and $E_{i}$ are pressure, temperature, ionization state, ion density, internal energy and ionization energy respectively. To quantify the uncertainty the predictions of three codes were compared: CASSANDRA, INFERNO [25] and NuQEOS. These are average atom codes which treat the electron equation of state with a fully quantum mechanical for bound electrons reverting to the semiclassical Thomas Fermi model for continuum electron states at different thresholds (CASSANDRA; INFERNO) or in a semi-classical Thomas-Fermi model for both bound and free electrons (NuQEOS). Comparison of INFERNO and NUQEOS for an iron plasma shows a divergence in predicted $Z^{*}$ at $150 \mathrm{mg} / \mathrm{cc}$ above $200 \mathrm{eV}$. This is shown in Fig 9. 


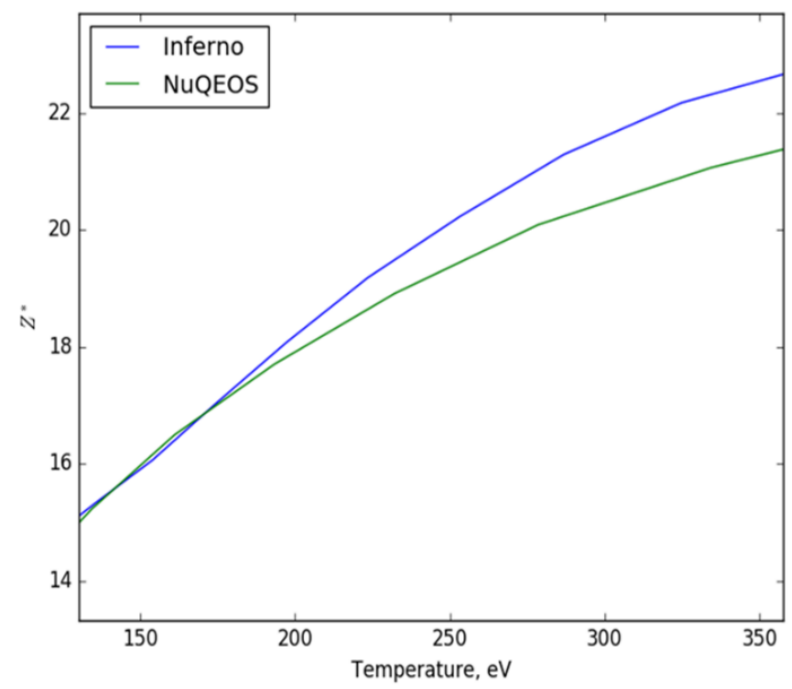

Fig 9: (Colour online) Comparison of mean ionization predicted by Inferno and NuQEOS.

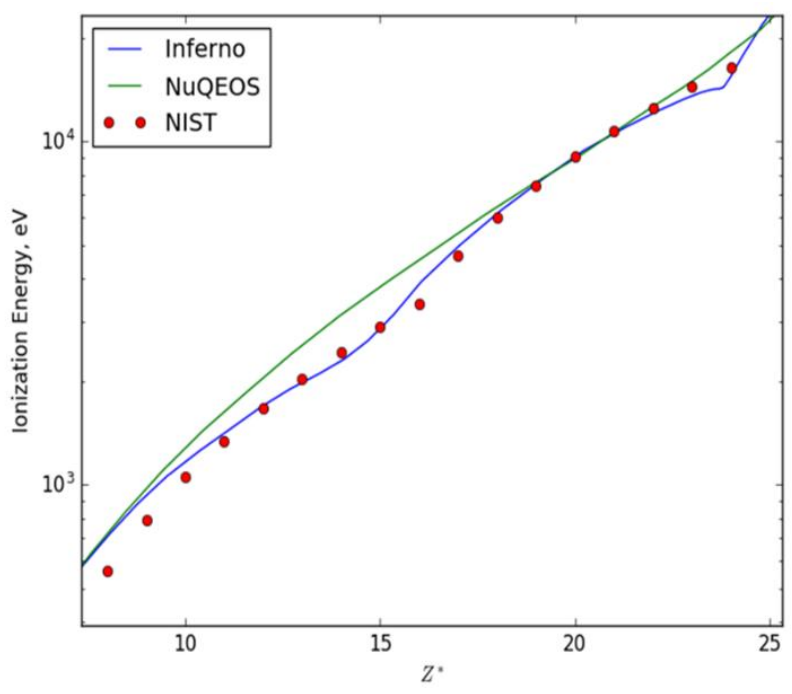

Fig 10 (colour online): Comparison of Inferno and NuQEOS predicted ionization energies compared to the NIST database.

One possible cause for the difference in the predicted mean ionization due to the two codes is the calculation of the ionization energy. Fig 10 shows a comparison of iron ionization energy calculated by Inferno and NuQEOS and compared against the NIST data base values. 


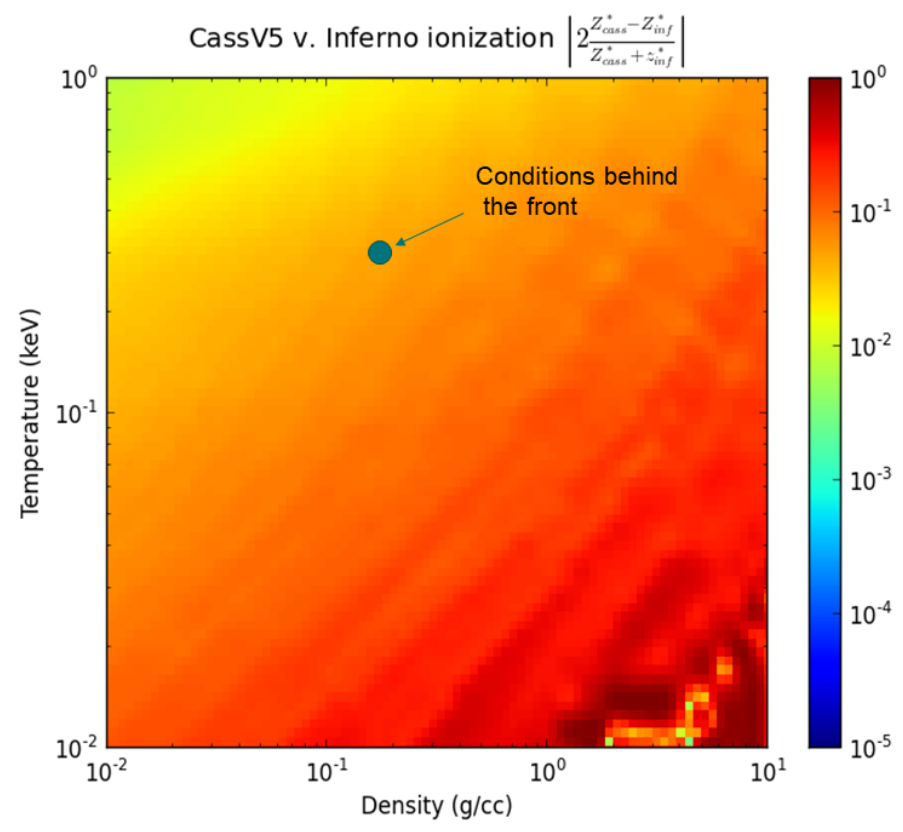

Fig 11: (colour online) CASSANDRA iron $Z^{*}$ compared to Inferno as the ratio of the difference over the average for temperature-density space around the experimental conditions. At the conditions in the experiment indicated on the figure, the difference is around $5 \%$.

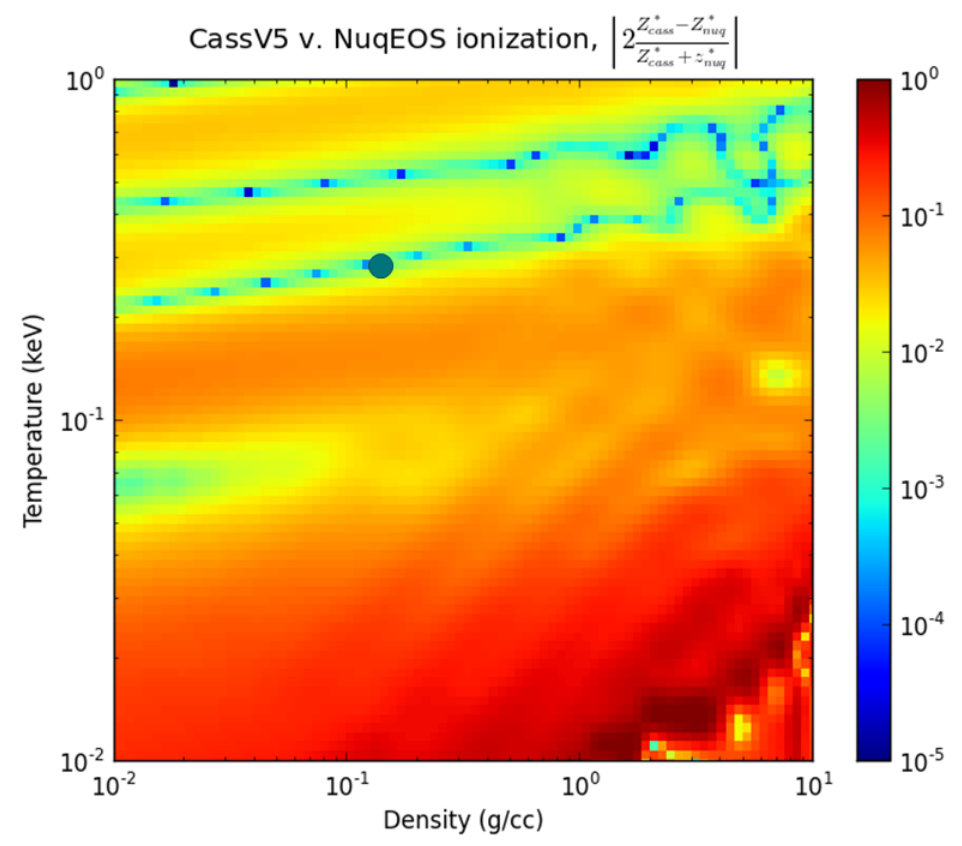

Fig 12: colour online CASSANDRA compared to NuQEOS. Differences are around a few percent at the conditions predicted behind the radiation wave-front. The banding seen is due to shell structure being absent in NuQEOS. 
Although some of the difference can be attributed to this it is not enough to account for the $Z^{*}$ variation seen in Fig 9. The models were then compared to the prediction of the CASSANDRA opacity code which has been extensively tested against experiment in the temperature-density conditions of the proposed experiment. The difference over the average was taken for $Z^{*}$ calculated for CASSANDRA and Inferno and CASSANDRA and NuQEOS. The results are shown in Figs 11 and 12 respectively. Over the conditions behind the front differences in $Z^{*}$ are in the 5\% range for Inferno and the few percent for NuQEOS which is the model used to generate the EoS in the predictions presented in this paper. That these different EoS models give results which only differ on the 5\% level gives confidence that the uncertainty in the EoS is not large enough to compromise the experiments. However, more work is needed to better understand the equation of state differences between codes and further work is underway using the OPENSESAME suite and particle swarm fitting methods [26, 27] for a range of equation of state calculations and measurements.

Referring to equation 1 it is possible to make an estimate of the accuracy possible in the burn-through experiment assuming the uncertainties given for the various parameters are as those given above, which are based on findings from previous NIF experiments using the same hohlraum and diagnostics. Taking the errors as adding in quadrature:-

$$
\left(\frac{\Delta \kappa}{\kappa}\right)^{2}=3 \cdot\left(\frac{\Delta T}{T}\right)^{2}+2 \cdot\left(\frac{\Delta \rho}{\rho}\right)^{2}+2 \cdot\left(\frac{\Delta x}{x}\right)^{2}+\left(\frac{\Delta t}{t}\right)^{2}+\left(\frac{\Delta C_{v}}{C_{v}}\right)^{2}
$$

Taking the uncertainties as radiation temperature $2.5 \%$ (for a $5 \%$ flux uncertainty); the density as $2 \%$; foam length as $3 \%$; timing uncertainty as $4 \%(100 \mathrm{ps}$ in $2.5 \mathrm{~ns})$ and a specific heat uncertainty of $5 \%$, the opacity uncertainty is around 9\%. Since the difference seen in the Sandia iron experiments is a factor of between $200 \%$ and $400 \%$ from current theory prediction, this estimate suggests that a burn-through measurement should be able to discriminate this difference, even if some of the uncertainties prove to be underestimates. Note that this assumes the increase in the opacity observed in Sandia iron experiments in the range $800-1300 \mathrm{eV}$ applies at lower frequencies which are also important in determining the radiation transport in iron at the conditions of these experiments and in the solar interior.

\section{SUMMARY/CONCLUSION}


A proposal for a radiation wave burn-through experiment in an iron oxide sample has been described to further investigate the opacity of iron at conditions close to the solar convective-radiative zone boundary. This is motivated by the recent findings of experiments at the Sandia National Laboratory $Z$ facility of a large enhancement, between a factor of 2 and 4 , in the opacity of iron at these conditions compared to theory. These experiments would be complementary to the work underway already on NIF to repeat the $\mathrm{Z}$ results using frequency-resolved X-ray spectroscopy [14]. The DANTE diagnostics on NIF will be used to measure the radiation wave transit time and the flux to compare to simulations where the iron opacity is the nominal value predicted by current theory and where the opacity is increased in line with the $\mathrm{Z}$ experimental observations. Simulated DANTE traces have been generated and used to predict the feasibility of the experiment based on sensitivity studies. The values of uncertainty bounds on the parameters determining the radiation wave transit have been based on measurements of previous NIF experiments using the same hohlraum drive and code comparisons for material properties. A simple error analysis suggests that even if these bounds have been underestimated, the opacity could still be measured accurately enough to discriminate between current theory predictions and an enhancement of a factor of two in opacity. Simulated DANTE traces

taking measurement errors into account show a measurable difference in emergent flux and radiation wave transit for a factor of 2 change in the iron opacity. More work is needed to reduce the error due to uncertainties on the material equation of state. The other error bounds are limited by the measurement techniques. It is planned to field radiation driven iron oxide experiments on the Orion and OMEGA facility at lower temperature to better understand the material properties of the foam samples ahead of experiments using NIF.

\section{AKNOWLEDGEMENTS}

The authors would like to acknowledge useful discussions with Dr Peter Hatfield on the implications of enhanced iron opacity for astroseismology and exoplanet searches. This paper is published under @ British Crown Owned Copyright 2019/AWE.

\section{REFERENCES}

1. M. Asplund, Annu. Rev. Astron. Astrophys. 43, 481 (2005)

2. C. Allende Prieto, D. L. Lambert and M. Asplund, Astrophys. J. 556, L63 (2001) 
3. M. Asplund et al., Annu. Rev. Astron. Astrophys. 47, 481, (2009)

4. S. Turck-Chièze et al, Phys. Rev. Lett. 93, 211102 (2004)

5. S. Basu and H. M. Antia, Phys. Rep. 457, 217 (2008)

6. J. N. Bahcall, A. M. Serenelli and M Pinsonneault, Astrophys. J. 614, 464 (2004)

7. H. M. Antia and S. Basu, Astrophys. J. 620, L129 (2005)

8. J. E. Bailey et al. Nature, 517, 56 (2015)

9. J. E. Bailey et al, Phys. Plasmas 16, 058101 (2009)

10. C. A. Iglesias, High Energy Density Phys. 15, 4 (2015)

11. E. Moraveji, MNRAS 000, arXiv:1509.08652v1 (2015)

12. P. Walczak, arXiv:1704.06067v1 (2017)

13. M. P. Di Mauro, arXiv:1703.07604v2 (2017)

14. T. S. Perry et al, High Energy Density Phys. 23, 223 (2017)

15. D. Mihalas, Stellar Atmospheres, W. H. Freeman and Co. (1978)

16. Ya. B. Zel'dovich and Yu. P. Raizer, Physics of Shock Waves and High Temperature Hydrodynamic Phenomena, Dover (2002)

17. A. S. Moore et al, J. Quant. Spectrosc. Radiat. Trans., 159, 19 (2015)

18. E. L. Dewald et al, Rev. Sci. Instrum. 75, 3759 (2004)

19. P. D. Roberts et al, J. Phys. D, 13, 1957 (1980)

20. C. C. Smith, High Energy Density Phys. 6, 48 (2010)

21. J. H. Hammer, M. D. Rosen, Phys. Plasmas, 10, 1829 (2003)

22. B. J. B. Crowley and J. W. Harris, J. Quant. Spectrosc. Radiat. Trans., 71, 257 (2001)

23. J. A. Fleck and J. D. Cummings, J. Comput. Phys. 8, 313 (1971)

24. S. D. Rothman et al, Phys. Plasmas, 11, 5620 (2004)

25. D. A. Liberman, Phys. Rev. B 20, 4981 (1979)

26. J. A. Gaffney et al, High Energy Density Phys. 28, 7 (2018)

27. J. F. Kennedy et al, Swarm Intelligence, Morgan Kaufmann (2001) 10.1016/B978-155860595-4/50000-0 